\title{
Avaliação Clínica Comparativa entre a Cetamina e a Clonidina por Via Peridural no Tratamento da Dor Crônica Neuropática *
}

\section{Epidural Ketamine Versus Epidural Clonidine as Therapeutic for Refractory Neuropathic Chronic Pain}

Gabriela Rocha Lauretti ${ }^{1}$, Alexandre de Menezes Rodrigues ${ }^{2}$, Josenília Maria Alves Gomes ${ }^{2}$, Marlene Paulino dos Reis, TSA ${ }^{4}$

\begin{abstract}
RESUMO
Lauretti GR, Rodrigues AM, Gomes JMA, Reis MP - Avaliação Clínica Comparativa entre a Cetamina e a Clonidina por Via Peridural no Tratamento da Dor Crônica Neuropática
\end{abstract}

Justificativa e Objetivos - A dor crônica resulta em resposta aumentada dos neurônios do corno dorsal da medula espinhal, devido à ação de diferentes mediadores liberados pelas terminações neuronais, incluindo agonistas para os receptores $\mathrm{N}$-metil-D-aspartato. Além das vias ascendentes sensitivas, vias descendentes inibitórias modulam a sensação de dor, incluindo a participação de $\alpha_{2}$-agonistas localizados nos aferentes primários e na medula espinal. Este estudo visou avaliar o efeito antinociceptivo da cetamina (antagonista do receptor $N$-metil-D-aspartato) e da clonidina ( $\alpha_{2}$ - agonista) administrada por via peridural, no tratamento da dor crônica neuropática.

Método - Vinte e seis pacientes adultos com queixa de dor tipo neuropática, não-responsiva aos tratamentos convencionais, foram de forma prospectiva, aleatória e duplamente encoberta, divididos em dois grupos. Todos os pacientes fizeram uso de 50-75 mg de amitriptilina por via oral diariamente. A dor foi avaliada utilizando-se a escala analógica visual (EAV) de 10 $\mathrm{cm}$, em que "zero" correspondeu à "ausência de dor" e "dez" à "pior dor imaginável". Um cateter peridural foi inserido na região lombar, e as medicações por via peridural foram administradas em intervalos de 8 horas, durante três semanas. O Grupo Cetamina recebeu 0,1 mg. $\mathrm{kg}^{-1}$ de cetamina sem conservante, seguida da administração de $30 \mathrm{mg}$ de lidocaína a $1 \%$, em cada aplicação. O Grupo Clonidina recebeu $30 \mu \mathrm{g}$ de clonidina sem conservante, seguida da administração de 30 mg de lidocaína a 1\%, em cada aplicação.

Resultados - Vinte e três pacientes fizeram parte da avaliação final. A administração de cetamina ou clonidina por via peridural, nas doses propostas, resultou em analgesia durante todo o período da manutenção do cateter peridural (EAV inicial

\footnotetext{
* Recebido da (Received from) Clínica para o Tratamento da Dor - Hospital das Clínicas da Faculdade de Medicina de Ribeirão Preto- Universidade de São Paulo (HC-FMRP-USP)

1. Professora Doutora do Departamento de Biomecânica, Medicina e Reabilitação do Aparelho Locomotor - FMRP - USP

2. ME em dor Dor no HC da FMRP - USP

3. Pós-graduanda do HC da FMRP - USP

4. Professora Associada do Departamento de Biomecânica, Medicina e Reabilitação do Aparelho Locomotor - FMRP - USP
}

Apresentado (Submitted) em 25 de maio de 2001

Aceito (Accepted) para publicação em 26 de julho de 2001

Correspondência para (Mail to):

Dra. Gabriela Rocha Lauretti

Rua Campos Sales, 330/44

14015-110 Ribeirão Preto, SP

(c) Sociedade Brasileira de Anestesiologia, 2002
8-10 cm versus EAV final $0-3 \mathrm{~cm})(p<0,002)$. Após a retirada do cateter peridural, os valores numéricos da EAV mantiveram-se entre $0-3 \mathrm{~cm}$ durante 2 a 5 semanas.

Conclusões - A administração de cetamina ou clonidina por via peridural resultou em ação antinociceptiva em pacientes com dor crônica neuropática, não-responsiva aos tratamentos convencionais, representando alternativas eficazes, quando o tratamento convencional não obteve sucesso.

UNITERMOS - ANALGÉSICOS: clonidina; DOR, Crônica: neuropática; HIPNÓTICOS: cetamina; TÉCNICAS ANESTÉSICAS, Regional: peridural contínua

\section{SUMMARY}

Lauretti GR, Rodrigues AM, Gomes JMA, Reis MP - Epidural Ketamine Versus Epidural Clonidine in the Treatment of Neuropathic Chronic Pain

Background and Objectives - Chronic pain results in an increased response of spinal cord dorsal horn neurons due to the action of several mediators released by neuronal terminals, including the agonists of $\mathrm{N}$-methyl-D-aspartate receptors. In addition to sensory ascending pathways, inhibitory descending pathways modulate pain, including $\alpha_{2}$-adrenoceptors located on primary afferent terminals and on the spinal cord. This study was designed to investigate the anti-noxious effects of epidural ketamine ( $N$-methyl-D-aspartate antagonist) or epidural clonidine $\left(\alpha_{2}\right.$-adrenergic agonist) in the treatment of neuropathic chronic pain.

Methods - Twenty-six adult patients, with neuropathic chronic pain not responsive to conservative therapy, were randomly divided into two groups in this prospective double-blind study. All patients were regularly taking $50-75 \mathrm{mg}$ oral amitriptyline at bedtime. Pain was evaluated through a $10 \mathrm{~cm}$ visual analog scale (VAS), with "zero" corresponding to "no pain" and 10 to "the worst possible pain". A lumbar epidural catheter was inserted and test drugs were administered at 8 hour-intervals during 3 weeks. The ketamine group (KG) was given each time 1 $\mathrm{mg} . \mathrm{kg}^{-1}$ preservative-free ketamine followed by $30 \mathrm{mg}$ of $1 \%$ lidocaine. The clonidine group (Clo G) was given $30 \mu \mathrm{g}$ preservative-free clonidine followed by $30 \mathrm{mg}$ of $1 \%$ lidocaine $(3 \mathrm{ml})$.

Results - Twenty-three patients were evaluated (KG- $n=10$; Clo $G-n=13)$. Epidural administration of ketamine or clonidine in the proposed doses resulted in analgesia during epidural catheter maintenance (initial VAS 8-10 cm versus final VAS $0-3 \mathrm{~cm})(p<$ $0.002)$. VAS scores remained maintained between 0 and $3 \mathrm{~cm}$ from 2 to 5 weeks following epidural catheter removal.

Conclusions - Epidural ketamine or clonidine resulted in analgesia for neuropathic chronic pain refractory to conservative treatment and are effective alternatives when conventional treatment fails.

KEY WORDS - ANALGESICS: clonidine; ANESTHETIC TECHNIQUES, Regional: epidural continuous; HYPNOTICS: ketamine; PAIN, Chronic: neuropathic 


\section{INTRODUÇÃO}

A causa clínica mais provável de dor neuropática é a lesão axonal aguda, associada a um processo de degeneração Walleriana, que por sua vez depende do recrutamento de macrófagos e da atividade das citocinas. A dor neuropática é transmitida através de fibras não-mielinizadas do tipo $C$ e do tipo $A \beta$, que transmitem a informação $a$ partir de mecanorreceptores de baixo limiar. As fibras $A \beta$ que normalmente fazem sinapse na lâmina III do corno posterior da medula espinhal, na dor neuropática interagem com as vias nociceptivas da lâmina II, com os neurônios de amplo padrão de resposta da lâmina $V$ e com as projeções tálamo-corticais ${ }^{1}$, desencadeando um processo de sensibilização central e alterações anatômicas na medula espinhal, correspondentes à doença dor crônica ${ }^{2}$.

Pacientes portadores da doença dor crônica neuropática freqüentemente não respondem aos tratamentos convencionais com antiinflamatórios não-esteroidais, antidepressivos, fisioterapia ou o opióide tramadol. A simpatectomia, na distribuição do dermátomo afetado, é algumas vezes efetiva em reduzir ou eliminar a dor, mas o resultado é apenas transitório, na maioria dos casos. Para os casos refratários aos tratamentos convencionais, há a possibilidade da administração de medicações por via invasiva, sendo que os $\alpha_{2}$-agonistas $^{3}$ e os antagonistas do complexo receptor N-metil-D-aspartato ${ }^{4}$ representam fármacos potencialmente úteis no controle da plasticidade da dor neuropática.

Este estudo visou avaliar a eficácia da cetamina (antagonista do receptor $\mathrm{N}$-metil-D-aspartato) e da clonidina ( $\alpha_{2}$-agonista), administradas por via peridural, no controle da dor neuropática crônica, refratária aos tratamentos convencionais.

\section{MÉTODO}

O estudo foi aprovado pela Comissão de Normas Éticas do Hospital das Clínicas da Faculdade de Medicina de Ribeirão Preto - USP, e o consentimento para participação foi obtido. Participaram do estudo 26 pacientes adultos, com idades entre 21 e 65 anos, portadores de dor crônica do tipo neuropática, com história de dor por tempo superior a seis meses de duração, não-responsiva ao tratamento com antiinflamatórios não-esteroidais, fisioterapia, antidepressivos, tramadol ou meperidina por via venosa. O estudo foi prospectivo, aleatório, duplamente encoberto e realizado ambulatorialmente. Aintensidade da dor foi avaliada utilizando-se a Escala Analógica Visual de $10 \mathrm{~cm}$ (EAV), sendo o extremo "zero cm" correspondente à "ausência de dor", variando até "dez cm", correspondente à "pior dor imaginável". Todos os pacientes fizeram uso de 50 a $75 \mathrm{mg}$ de amitriptilina por via oral, diariamente durante todo o período do tratamento.

Vinte e seis pacientes foram designados a um de dois grupos. Estando em uso de 50 a 75 mg de amitriptilina diariamente por pelo menos 7 dias, foi instalado um cateter peridural na região lombar, utilizando-se técnica asséptica e filtro antibacteriano. $\mathrm{O}$ correto posicionamento do cateter foi veri- ficado com a administração de $3 \mathrm{ml}$ de lidocaína a 1\%, após a realização da dose teste com $3 \mathrm{ml}$ de lidocaína a $1 \%$, com vasoconstritor (volume total $6 \mathrm{ml}$ ). O Grupo Cetamina recebeu $0,1 \mathrm{mg} \cdot \mathrm{kg}^{-1}$ de cetamina racêmica sem conservante $(2 \mathrm{ml})$, diluída em solução de lidocaína a $1 \%$ sem vasoconstritor, seguida da administração de 30 mg de lidocaína a $1 \%$ sem vasoconstritor ( $3 \mathrm{ml}$ ). Esta aplicação foi realizada diariamente, a intervalos de 8 horas, pelo próprio paciente, sendo a dose total diária de cetamina $0,3 \mathrm{mg} \cdot \mathrm{kg}^{-1}$.dia. O Grupo Clonidina recebeu $30 \mu \mathrm{g}$ de clonidina sem conservante $(2 \mathrm{ml})$, diluída em solução de lidocaína a $1 \%$ sem vasoconstritor, seguida da administração de $30 \mathrm{mg}$ de lidocaína a $1 \%$ sem vasoconstritor (3 ml). Esta aplicação foi realizada diariamente a intervalos de 8 horas, pelo próprio paciente, sendo a dose total diária de clonidina $90 \mu \mathrm{g}$. O cateter foi mantido por 3 semanas consecutivas, sendo que os pacientes tinham retornos semanais para troca de curativo, avaliação da dor, avaliação dos efeitos adversos, e fornecimento dos fármacos testes e de lidocaína a 1\%. Os pacientes receberam semanalmente os frascos de lidocaína a $1 \%$ com as drogas testes preparadas por um observador independente, sendo $2 \mathrm{ml}$ o volume a ser administrado, seguido da administração de $3 \mathrm{ml}$ de lidocaína a $1 \%$.

Após 3 semanas consecutivas de avaliação, o cateter peridural foi retirado, sendo mantida a dose de amitriptilina por via oral. Foram prescritos para os pacientes $50 \mathrm{mg}$ de cetoprofeno por via oral, a intervalos mínimos diários de 8 horas, se necessário. O consumo de cetoprofeno foi avaliado após a retirada do cateter peridural, durante o tempo em que o paciente apresentava valor numérico menor que $4 \mathrm{~cm}$ pela EAV $(0-10 \mathrm{~cm})$.

O número mínimo de pacientes determinado pelo teste do poder estatístico é 8 , e foi baseado em estudo preliminar realizado em nosso serviço, em que diferentes doses foram testadas previamente, considerando alfa $=5 \%$ e beta $=0,1$. Os grupos foram comparados para análise demográfica pelo teste de Mann Whitney. Aincidência dos efeitos adversos e o sexo dos pacientes foram comparados entre os grupos, utilizando-se o teste Qui-quadrado. Os valores numéricos da EAV-10 cm semanais de um mesmo grupo foram avaliados pelo teste Friedman ANOVA, seguido pelo teste Wilcoxon matched pareado. Os valores numéricos da EAV- $10 \mathrm{~cm}$ entre os grupos foram comparados pelo teste MANOVA, seguido do teste "Tukey Honest significance". Os dados são expressos como média \pm desvio padrão, sendo que $p<0,05$ foi considerado significante.

\section{RESULTADOS}

Vinte e três pacientes fizeram parte da análise final (grupo Cetamina- $n=10$; grupo Clonidina- $n=13$ ). Um dos pacientes do grupo Cetamina foi excluído porque queixou-se de tontura e recusou-se a continuar o estudo. Outro paciente foi excluído porque procurou o serviço com queixa de parestesia em um dos membros inferiores no segundo dia de avaliação, sendo constatado mal posicionamento do cateter peridural, 
sendo o mesmo retirado. Outro paciente foi excluído devido à obtenção de dados de forma incompleta.

Os grupos foram demograficamente semelhantes. Todos os pacientes eram portadores de dor crônica, do tipo neuropática, e foram classificados como estado físico ASAII. No grupo cetamina, 6 pacientes eram do sexo masculino, e 4 do sexo feminino. No grupo clonidina, 8 pacientes eram do sexo masculino, e 5 eram do sexo feminino $(p=0,999)$. Os grupos foram semelhantes quanto à idade (anos) (grupo cetamina versus grupo clonidina - $46 \pm 12 ; 47 \pm 10 ; p=0,2414$ ); quanto ao peso corporal $(\mathrm{kg})$ (grupo cetamina versus grupo clonidina $-63 \pm 13 ; 63 \pm 8 ; p=0,8947$ ) e quanto à estatura $(\mathrm{cm})$ (grupo cetamina versus grupo clonidina- $165 \pm 8 ; 162 \pm 6 ; p=$ $0,9322)$.

O valor numérico classificado pela EAV $(0-10 \mathrm{~cm})$ está descrito na figura 1 . Os valores iniciais, antes de se iniciar o tratamento com o cateter peridural foram semelhantes entre os dois grupos e variaram entre 7 e $10 \mathrm{~cm}(p=0,5708)$. Os valores da EAV $(0-10 \mathrm{~cm}) 7,14$ e 21 dias após a instalação do cateter peridural variaram entre 0 e $3 \mathrm{~cm}$ e foram semelhantes quando comparados entre os dois grupos em um mesmo tempo (com 7 dias: $p=0,4611 ; 14$ dias: $p=0,2414 ; \mathrm{e} 21$ dias: $p$ $=0,8947$ ). Entretanto, os valores numéricos em um mesmo grupo foram estatisticamente diferentes quando comparados ao valor de EAV inicial, com o obtido com 7 dias $(p=$ $0,0014), 14$ dias $(p=0,0014)$ e 21 dias $(p=0,0014)$. Um paciente de cada grupo referiu dor na pele e tecido subcutâneo, na área ao redor da inserção do cateter peridural, po-

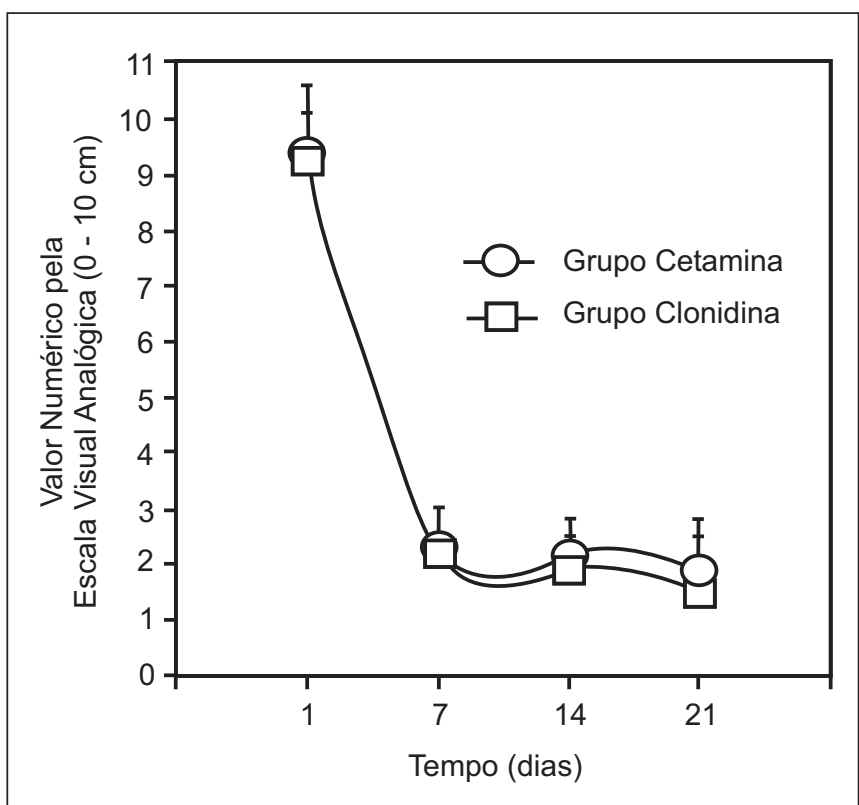

Figura 1 - Valores Numéricos pela Escala Analógica Visual de 0-10 cm nos períodos: inicial (dia 1); sétimo dia, décimo quarto dia e vigésimo primeiro dia após a instalação do cateter peridural. Os valores da EAV $(0-10 \mathrm{~cm})$ foram semelhantes quando comparados entre os dois grupos em um mesmo tempo (com 7 dias- $p=0,4611 ; 14$ dias- $p=0,2414$; e 21 dias- $p=0,8947)$. Os valores da EAV em um mesmo grupo foram diferentes quando comparados o valor de EAV inicial, com o obtido com 7 dias $(p=0,0014), 14$ dias $(p=0,0014)$ e 21 dias $(p=0,0014)$ rém não havia sinal aparente de infecção local, e os curativos foram realizados semanalmente, seguindo o protocolo. Dois pacientes do grupo Clonidina (que eram hipertensos, porém controlados), apresentaram hipotensão leve após a administração da clonidina por via peridural, sendo observados e tendo-Ihes sido recomendado ficarem em repouso após cada administração da medicação peridural por 30 a 40 minutos. Não foram observados outros efeitos adversos.

Um paciente do grupo cetamina queixou-se de parestesia no $14^{\circ}$ dia de avaliação, e o cateter peridural foi retirado com regressão espontânea do quadro. Dois pacientes do grupo clonidina queixaram-se de dor à injeção da droga teste peridural no $14^{\circ}$ dia de avaliação, e o cateter peridural foi removido. Em dois dos pacientes que utilizaram cetamina por via peridural, e um dos pacientes que utilizou clonidina por via peridural, foi posteriormente diagnosticada neoplasia (fígado, intestino e linfoma; respectivamente). Posteriormente ao diagnóstico, o cateter peridural foi recolocado nestes pacientes, e iniciada morfina (4 a 8 mg.dia) por via peridural, diariamente. Dois dos pacientes foram a óbito no período de $8 \mathrm{me}-$ ses após o diagnóstico.

Todos os pacientes mantiveram valores numéricos classificados pela EAV $(0-10 \mathrm{~cm})$ entre 0 e $3 \mathrm{~cm}$, durante 2 a 5 semanas após a retirada do cateter peridural. O consumo de cetoprofeno durante este período de observação foi esporádico entre os pacientes (variando de 1 comprimido a cada três dias a 2 comprimidos diários), e semelhante entre os grupos $(p=0,4929)$.

\section{DISCUSSÃo}

Os resultados obtidos demonstraram que a administração diária de baixas doses de clonidina (90 $\mu$ g.dia) ou cetamina (0,3 mg. $\mathrm{kg}^{-1}$.dia) associadas à lidocaína, por via peridural, resultou em controle da dor neuropática crônica, refratária aos tratamentos convencionais. Nas doses utilizadas, os pacientes tiveram condições de realizar o tratamento de forma ambulatorial, sendo o tratamento proposto bem tolerado, eficiente quanto à ação analgésica, e sem efeitos adversos limitantes.

A cetamina, um dos fármacos utilizados, apresenta um potencial terapêutico no tratamento da alodinia e hiperalgesia, componentes da dor neuropática ${ }^{5}$. As doses de cetamina descritas na literatura, administradas por via peridural, variam de 0,2 a $1 \mathrm{mg} \cdot \mathrm{kg}^{-16,7}$. A dose utilizada no estudo proposto foi de $0,3 \mathrm{mg} \cdot \mathrm{kg}^{-1}$. dia, dividido em três doses diárias, o que provavelmente contribuiu para a pequena incidência de efeitos adversos.

A ação analgésica da cetamina administrada por via espinhal é secundária ao bloqueio da condução axonal ${ }^{8}$ e da modulação da plasticidade central, inibindo a sensibilização central ${ }^{9}$. A cetamina interage com o local de ligação fenciclidina, associado ao complexo receptor $\mathrm{N}$-metil-D-aspartato através de dois mecanismos distintos: 1) parece exercer seu efeito analgésico com o canal acoplado ao complexo recep- 
tor $\mathrm{N}$-methil-D-aspartato aberto ${ }^{10}$, reduzindo o tempo de abertura do mesmo através de ligação em local de ação localizado dentro do canal ${ }^{11}$;2) diminui a freqüência de abertura do canal acoplado ao receptor N-metil-D-aspartato através de um mecanismo de ação alostérico, o qual envolve um local de ação localizado na região hidrofóbica da membrana, externamente ao canal, não necessitando necessariamente que o canal acoplado esteja em sua forma aberta ${ }^{11}$. Além da atividade relacionada ao complexo $\mathrm{N}$-metil-D-aspartato, possui atividade em receptores opióides, quisqualato, muscarínicos, monoaminérgicos e em canais de $\mathrm{Ca}^{++}$voltagem-dependente ${ }^{12-16}$.

O outro fármaco analgésico utilizado neste estudo, e que demonstrou potencial analgésico quando administrado em baixas doses ( $90 \mu$ g.dia), foi a clonidina. As doses de clonidina administradas por via peridural descritas na literatura variam de 1 a $8 \mu \mathrm{g} . \mathrm{kg}^{-1}$ administradas em bolus somente, ou seguidas de infusões contínuas $\left(0,5 \text { a } 2 \mu \mathrm{g} \cdot \mathrm{kg}^{-1} \cdot \mathrm{h}^{-1}\right)^{7,17,18}$. Quando administrada por via espinhal, a clonidina foi eficaz para a alodinia ${ }^{19}$ e para a dor crônica neoplásica refratária à morfina, particularmente do tipo neuropático, em que os pacientes receberam infusão contínua de clonidina, $30 \mu \mathrm{g} \cdot \mathrm{h}^{-13}$. $\mathrm{O}$ alívio da hiperalgesia seria devido à ação em receptores $\alpha_{2}$-adrenérgicos pré-sinápticos através da inibição da liberação de noradrenalina de neurônios simpáticos pós-ganglionares ${ }^{20}$. Em pacientes com história de distrofia simpática reflexa, $300 \mu \mathrm{g}$ ou $700 \mu \mathrm{g}$ de clonidina administrada por via peridural foi eficaz para o controle da mesma ${ }^{21}$.

Aclonidina, um agonista $\alpha_{2}$-adrenérgico parcial, age não somente em receptores $\alpha_{2}$-adrenérgicos mas em receptores $\alpha_{1}$-adrenérgicos e em receptores imidazolina, sendo a seletividade para receptores $\alpha_{2 \mathrm{~A}} / \alpha_{1}$ igual a $39^{22}$. A seletividade para receptores $\alpha_{2 A}$ /imidazolina é 16 . Sua ação analgésica espinhal é mediada em parte pela ativação colinérgica e estimulação da liberação de norepinefrina na medula ${ }^{23}$. No homem, a distribuição de receptores adrenérgicos localizados na medula espinhal varia, dependendo do nível. São mais abundantes na região sacral e cervical, comparados às regiões lombar e torácica ${ }^{24}$. Os subtipos $\alpha_{2 A}$ e $\alpha_{2 B}$ predominam na medula espinhal do homem, comparados ao subtipo $\alpha_{2 C}$ ${ }^{25}$, enquanto os subtipos de receptores $\alpha_{2 B}$ e $\alpha_{2 C}$ predominam no gânglio da raiz dorsal e também parecem participar da ação analgésica final da clonidina ${ }^{26}$.

No trabalho realizado, tanto a cetamina quanto a clonidina foram associadas ao anestésico local lidocaína, administrados por via peridural. A ação analgésica de baixas doses de lidocaína, na medula espinhal, difere de sua ação analgésica periférica. A lidocaína é mais potente no nível espinhal do que no periférico. Na periferia, interage com canais de $\mathrm{Na}^{+}$dependentes de voltagem, nolado intracelular da membrana, apresentando maior afinidade para o estado iônico aberto, gerado durante a despolarização. Além do mecanismo de ação descrito na medula espinhal, reduz direta ou indiretamente a despolarização pós-sináptica, mediada por receptores $\mathrm{N}$-metil-D-aspartato, e receptores para a neuroquinina e age, possivelmente, em canais de $\mathrm{Na}^{+}$, resistentes à tetrodotoxina. Possui ação colinomi- mética, nos receptores muscarínicos, e ativa os receptores sensitivos à glicina ${ }^{27-29}$.

Em conclusão, a administração peridural de $0,3 \mathrm{mg} \cdot \mathrm{kg}^{-1} \cdot$ dia de cetamina ou de $90 \mu \mathrm{g}$. dia de clonidina associadas à lidocaína, resultou em ação antinociceptiva em pacientes com dor crônica neuropática, não-responsiva aos tratamentos convencionais, por provável ação antinociceptiva central, representando alternativas eficazes, quando o tratamento convencional não obteve sucesso.

\section{Epidural Ketamine Versus Epidural Clonidine in the Treatment of Neuropathic Chronic Pain}

Gabriela Rocha Lauretti, M.D., Alexandre de Menezes Rodrigues, M.D., Josenília Maria Alves Gomes, M.D., Marlene Paulino dos Reis, TSA, M.D.

\section{INTRODUCTION}

The most likely clinical cause of neuropathic pain is an acute axonal injury associated to a Wallerian degeneration process which, in turn, depends on macrophages recruitment and cytokines activity. Neuropathic pain is transmitted through non-mielinated $C$ and $A \beta$ fibers, with information coming from low threshold mechanoreceptors. A $\beta$ fibers, which under normal conditions synapse in layer III of spinal cord posterior horn, may interact with layer II noxious ways, with layer $V$ highly responsive neurons and with thalamus-cortical projections in the presence of neuropathic pain ${ }^{1}$, triggering a central sensitization process and anatomic spinal cord changes which characterize the chronic pain disease ${ }^{2}$.

Neuropathic chronic pain patients often do not respond to conservative treatment with non-steroid anti-inflammatory drugs, antidepressants, physiotherapy or tramadol. Sympathectomy in the affected dermatome distribution is some times effective in reducing or eliminating pain, but this is mostly a transient result. For refractory cases, there is the possibility of invasive drug administration, being the $\alpha_{2}$ agonists ${ }^{3}$ and N-methyl-D-aspartate receptor antagonists ${ }^{4}$ potentially effective drugs to control neuropathic pain plasticity.

This study aimed at evaluating the efficacy of epidural ketamine (N-methyl-D-aspartate receptor antagonist) and clonidine ( $\alpha_{2}$-agonist) in controlling chronic neuropathic pain refractory to conservative treatment.

\section{METHODS}

After the Medical Ethics Committee approval and patients informed consent, 26 adult patients aged between 21 and 65 years, with neuropathic chronic pain for more then six months, refractory to NSAID, physiotherapy, antidepressants, tramadol or intravenous meperidine, were included in 
this double-blind prospective study. Pain intensity was evaluated by a $10 \mathrm{~cm}$ Visual Analog Scale (VAS) with "zero" corresponding "no pain" and "ten" to "the worst possible pain". All patients were receiving daily 50 to $75 \mathrm{mg}$ oral amitriptyline for at least 7 days before applying the study, which were kept throughout the treatment.

Patients were randomly distributed into two groups. Alumbar epidural catheter was inserted aseptically and an antibacterial filter installed. Catheter position was checked with the administration of $3 \mathrm{ml}$ of $1 \%$ lidocaine after a test dose of $3 \mathrm{ml} 1 \%$ lidocaine with vasoconstrictor (total volume of $6 \mathrm{ml}$ ). The Ketamine Group was given $0.1 \mathrm{mg} . \mathrm{kg}^{-1}$ preservative-free racemic ketamine $(2 \mathrm{ml})$ in $1 \%$ lidocaine without vasoconstrictor solution, followed by $30 \mathrm{mg}$ of $1 \%$ lidocaine without vasoconstrictor $(3 \mathrm{ml})$. Drugs were administered by the patients at 8-hour intervals in a total ketamine dose of $0.3 \mathrm{mg} . \mathrm{kg}^{-1}$.day. The Clonidine Group was given $30 \mu \mathrm{g}$ preservative-free clonidine $(2 \mathrm{ml})$ in $1 \%$ lidocaine without vasoconstrictor solution followed by $30 \mathrm{mg}$ of $1 \%$ lidocaine without vasoconstrictor ( 3 $\mathrm{ml}$ ). Drugs were administered by the patients at 8-hour intervals in a total daily clonidine dose of $90 \mu \mathrm{g}$. The catheter was maintained for 3 consecutive weeks. Patients would return once a week to change dressings, evaluate pain and adverse-effects and to be supplied with test drug and $1 \%$ lidocaine. Patients would received weekly the $1 \%$ lidocaine vials with the test drugs prepared by an independent observer, being 2 $\mathrm{ml}$ the volume to be administered followed by $3 \mathrm{ml}$ of $1 \%$ lidocaine.

After three consecutive weeks, epidural catheter was removed and oral amitriptyline was maintained. Oral $50 \mathrm{mg}$ ketoprofene at minimum 8-hour intervals was prescribed, if needed. Ketoprofene consumption was evaluated after epidural catheter removal during the period when patients presented below pain score $4 \mathrm{~cm}$ in the VAS scale $(0-10 \mathrm{~cm})$.

The smallest number of patients determined by the statistical power test for this study was 8 , based on a preliminary study performed in our hospital where different doses were previously tested considering alpha $=5 \%$ and beta $=0.1$. Mann Whitney test was used for demographic comparison. Chi-square test was used to compare adverse effects and gender between groups. Friedman ANOVA, followed by matched paired Wilcoxon test were used to evaluate VAS-10 weekly values in the same group. MANOVA test followed by Tukey Honest significance test were used to compare VAS-10 values between groups. Data are expressed in mean \pm standard deviation, considering significant $p<0.05$.

\section{RESULTS}

Twenty-three patients were included in the final analysis (Ketamine group $n=10$; Clonidine group $n=13$ ). One ketamine group patient was excluded for referring dizziness and refusing to continue with the study. Another one patient was excluded for referring lower limb paresthesia in the second evaluation day; epidural catheter malpositioning was confirmed and it was then removed. A third ketamine group patient was also excluded due to incomplete data report.
Groups were demographically similar. All patients referred neuropathic chronic pain and were classified as physical status ASA II. In the ketamine group, 6 patients were male and 4 female. In the clonidine group, 8 patients were male and 5 female $(p=0.999)$. Groups were similar in age (years) (ketamine group versus clonidine group - $46 \pm 12 ; 47 \pm 10 ; p=$ 0.2414 ), weight ( $\mathrm{kg}$ ) (ketamine group versus clonidine group $-63 \pm 13 ; 63 \pm 8 ; p=0.8947$ ) and height $(\mathrm{cm})$ (ketamine group versus clonidine group - $165 \pm 8 ; 162 \pm 6 ; p=0.9322$ ).

VAS $(0-10 \mathrm{~cm})$ values are shown in figure 1. Pretreatment values were similar to both groups and varied from 7 to $10 \mathrm{~cm} \mathrm{(p}$ $=0.5708)$. VAS $(0.10 \mathrm{~cm})$ values on the $7^{\text {th }}, 14^{\text {th }}$, and $21^{\text {st }}$ day after epidural catheter insertion varied from 0 to $3 \mathrm{~cm}$ and were similar when compared between groups in the same moment $\left(7^{\text {th }}\right.$ day: $p=0.4611 ; 14^{\text {th }}$ day: $p=0.2414$; and $21^{\text {st }}$ day: $p=0.8947)$. However, within each groups there were statistical differences when comparing initial VAS values to those obtained in day $7(p=0.0014)$, day $14(p=0.0014)$ and day $21(p=0.0014)$. One patient in each group referred skin and subcutaneous tissue pain around epidural catheter, but there was no evidence of local infection and dressings were weekly changed according to the protocol. Two clonidine group patients (hypertensive, however controlled), presented mild hypotension after epidural clonidine. They were observed and recommended to rest for 30 to 40 minutes after each epidural administration. There was no other adverse effect.

One ketamine group patient referred paresthesia in the $14^{\text {th }}$ evaluation day. The epidural catheter was removed, with

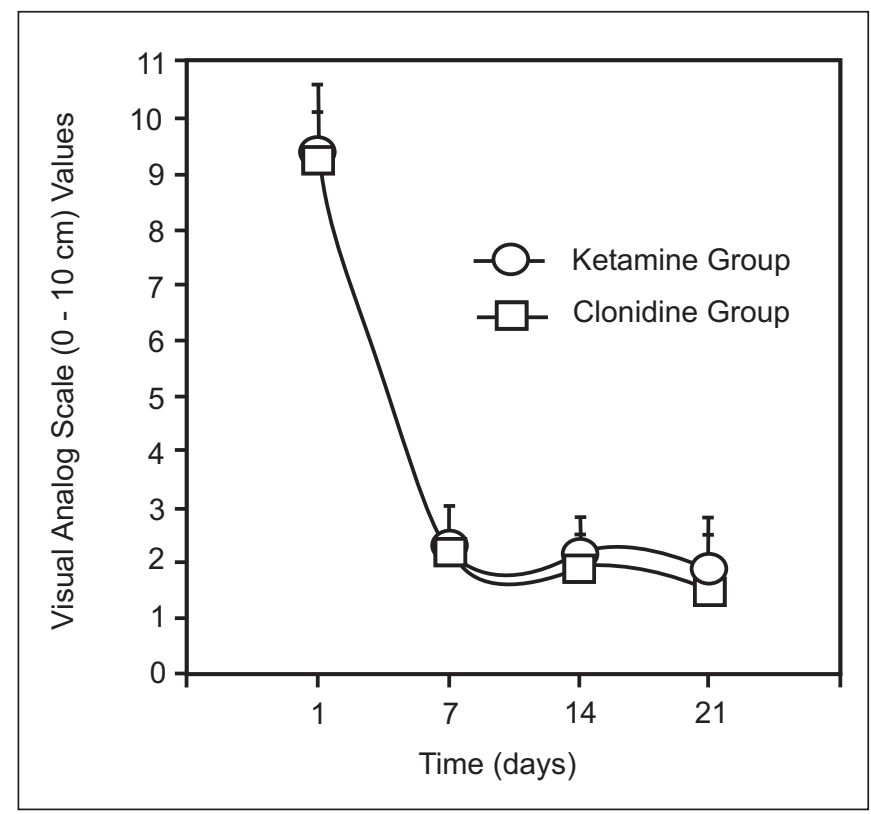

Figure 1 - Visual Analog Scale from 0-10 cm Values in the periods: initial (day 1); day 7, day 14 and day 21 after epidural catheter insertion. VAS $(0-10 \mathrm{~cm})$ values were similar when compared between groups in a same moment $\left(7^{\text {th }}\right.$ day- $p=0.4611 ; 14^{\text {th }}$ day- $p=0.2414$; and $21^{\text {st }}$ day- $p=0.8947$ ). VAS values for a same group were different when comparing initial VAS values with those obtained after 7 days $(p=0.0014), 14$ days $(p=0.0014)$ and 21 days $(p=0.0014)$

Revista Brasileira de Anestesiologia Vol. 52, No 1, Janeiro - Fevereiro, 2002 
spontaneous reversion of symptoms. Two clonidine group patients referred pain on injection in the $14^{\text {th }}$ evaluation day, and the epidural catheter was also removed. In two ketamine group patients and one clonidine group patient, neoplasia was posteriorly diagnosed (liver, intestine and lymphoma, respectively). After that, the epidural catheter was reinserted and daily epidural morphine was started ( 4 to $8 \mathrm{mg}$. day). Two patients died in an 8-month period after diagnosis.

All patients maintained VAS $(0-10 \mathrm{~cm})$ values between 0 and $3 \mathrm{~cm}$ during 2 to 5 weeks after epidural catheter removal. Ketoprofene consumption during this period was sporadic among patients (varying from 1 tablet every 3 days to 2 daily tablets) and similar between groups ( $p=0.4929)$.

\section{DISCUSSION}

Results have shown that the daily administration of low dose epidural clonidine ( $90 \mu \mathrm{g}$.day) or ketamine $\left(0.3 \mathrm{mg} \cdot \mathrm{kg}^{-1}\right.$.day) associated to lidocaine was able to control neuropathic chronic pain refractory to conservative treatment. In the doses used, individuals could be submitted to an outpatient regimen of treatment. The proposed approach was well tolerated, effective as to the analgesic action and without major side-effects.

Ketamine has a therapeutic potential in treating alodinia and hyperalgesia, which are neuropathic pain components ${ }^{5}$. Epidural ketamine doses described in the literature vary from 0.2 to $1 \mathrm{mg} \cdot \mathrm{kg}^{-16,7}$. Our proposed dose was $0.3 \mathrm{mg} \cdot \mathrm{kg}^{-1}$ divided in three daily administration, which has probably contributed to the low incidence of side-effects.

Spinal ketamine analgesic action is secondary to the axonal conduction block ${ }^{8}$ and central plasticity modulation, inhibiting central sensitization ${ }^{9}$. Ketamine interacts with fencyclidine binding site associated to $\mathrm{N}$-methyl-D-aspartate receptor complex through two different mechanisms: 1) it seems to produce the analgesic effect with the channel coupled to the open $\mathrm{N}$-methyl-D-aspartate receptor complex ${ }^{10}$ thus decreasing its opening time through binding to an action site located within the channel ${ }^{11} ; 2$ ) it decreases the opening frequency of the channel coupled to the N-methyl-D-aspartate receptor through an allosteric action mechanism involving an action site located in the membrane's hydrophobic region, externally to the channel, not necessarily needing the coupled channel to be in its open form ${ }^{11}$. In addition to $\mathrm{N}$-methyl-D-aspartate complex-related activity, it also acts on opioid receptors, quisqualates, muscarinics, monoaminergics and in voltage-dependent $\mathrm{Ca}^{++}$channels ${ }^{12-16}$.

The other studied drug which showed analgesic property when administered in low doses ( $90 \mu$ g.day) was clonidine. Epidural clonidine doses described in the literature vary from 1 to $8 \mu \mathrm{g} . \mathrm{kg}$ in bolus, that may be followed by continuous infusions $\left(0.5 \text { to } 2 \mu \mathrm{g} \cdot \mathrm{kg}^{-1} \cdot \mathrm{h}^{-1}\right)^{7,17,18}$. When spinally administered, clonidine was effective for alodinia ${ }^{19}$ and neoplasic chronic pain refractory to morphine, especially neuropathic pain, when patients received $30 \mu \mathrm{g} \cdot \mathrm{h}^{-1}$ clonidine continuous infusion. Hyperalgesia inhibition would be due its action on pre-synaptic $\alpha_{2}$-adrenergic receptors, reducing norepineph- rine release by post-ganglionary sympathetic neurons ${ }^{20}$. Epidural clonidine in a dose range of 300 to $700 \mu \mathrm{g}$ was effective for controlling reflex sympathetic distrophy ${ }^{21}$.

Clonidine, a partial $\alpha_{2}$-adrenergic agonist, acts not only in $\alpha_{2}$-adrenergic receptors but also in imidazoline receptors, with a selectivity ratio $\alpha_{2 A} / \alpha_{1}$ equal to $39^{22}$. The selectivity for $\alpha_{2 A}$ /imidazoline receptors is 16 . Its spinal analgesic action is partially mediated by cholinergic activation and stimulation of norepinephrine release in the spinal cord ${ }^{23}$. In humans, the adrenergic receptors amount varies along the spinal cord. They are more abundant at the sacral and cervical levels, comparing to the toracic and lumbar regions ${ }^{24}$.

Subtypes $\alpha_{2 A}$ and $\alpha_{2 B}$ are found in much greater amount than $\alpha_{2 C}$ subtype ${ }^{25}$, while $\alpha_{2 B}$ and $\alpha_{2 C}$ subtypes are predominant in dorsal root ganglion and seem to take part in the final analgesic action of clonidine ${ }^{26}$.

In our study, both epidural ketamine and clonidine were associated to lidocaine. The analgesic action of low doses of lidocaine in the spinal cord is different from its peripheral action. Lidocaine is more potent in the spine than in the periphery. In peripheral sities, it interacts with voltage-dependent $\mathrm{Na}^{+}$ channels in the membrane's intracellular showing more affinity to their open ionic state present during depolarization. In the spinal cord, besides the above described action, it directly or indirectly decreases post-synaptic depolarization mediated by $\mathrm{N}$-methyl-D-aspartate receptors and neuroquinine receptors, as well as possibly showing effect on $\mathrm{Na}^{+}$ channels resistant to tetrodotoxin. It has a cholinomimetic action on muscarinic receptors and activates glycine-sensitive receptors ${ }^{27-29}$.

In conclusion, epidural $0.3 \mathrm{mg} \cdot \mathrm{kg}^{-1}$.day ketamine or 90 $\mu \mathrm{g}$.day clonidine associated to lidocaine resulted in analgesic action for patients with neuropathic chronic pain non responsive to conservative treatment due to a possible central anti-noxious action therefore, they represent effective alternatives when conservative treatment fails.

\section{REFERÊNCIAS - REFERENCES}

01. Wall PD, Devor M - Sensory afferent impulses originate from dorsal root ganglia as well as from the periphery in normal and nerve injured rats. Pain, 1983;17:321-339.

02. Cousins MJ - Pain: the past, present and future of Anesthesiology? Anesthesiology, 1999;91:538-551.

03. Eisenach JC, DuPen S, Dubois M et al - Peridural clonidine analgesia for intractable cancer pain. Pain, 1995;61:391-399.

04. Lauretti GR, Gomes JMA, Reis MP et al - Low doses of peridural ketamine or neostigmine, but not midazolam, improve morphine analgesia in peridural terminal cancer pain therapy. J Clin Anesth, 1999,11:663-668.

05. Persson J, Axelsson G, Hallin RG et al - Beneficial effects of ketamine in a chronic pain state with allodynia, possibly due to a central sensitization. Pain, 1995;60:217-222.

06. Findlow D, Aldridge LM, Doyle E - Comparison of caudal block using bupivacaine and ketamine with ilioinguinal nerve block for orchidopexy in children. Anaesthesia, 1997;52:1090-1110. 
07. Cook B, Grubb DJ, Aldridge LA et al - Comparison of the effects of adrenaline, clonidine and ketamine on the duration of caudal analgesia produced by bupivacaine in children. $\mathrm{Br} \mathrm{J}$ Anaesth, 1995;75:698-701.

08. Iida H, Dohi S, Tanahashi T et al - Spinal conduction block by intrathecal ketamine in dogs. Anesth Analg, 1997;85:106-110.

09. Pocket S - Spinal cord synaptic plasticity and chronic pain. Anesth Analg, 1995;80:173-179.

10. Ghorpade A, Advokat C - Evidence of a role for N-methyl-D-aspartate (NMDA) receptors in the facilitation of tail withdrawal after spinal transection. Pharmacol Bioch Behav, 1994;48: 175-181.

11. Orser BA, Pennefather PS, MacDonald JF - Multiple mechanisms of ketamine blockade of $\mathrm{N}$-methyl-D-aspartate receptors. Anesthesiology, 1997;86:903-917.

12. Hurstveit $O$, Maurset $A$, Oye I - Interaction of the chiral forms of ketamine with opioid, phenciclidine, and muscarinic receptors. Pharmacol Toxicol, 1995;77:355-359.

13. Ashford ML, Boden P, Ramsey RL et al - Enhancement of desensitization of quisqualate-type glutamate receptor by the dissociative anaesthetic ketamine. J Exp Biol, 1989;141:73-86.

14. Crisp T, Perroti JM, Smith DL et al - The local monoaminergic dependence of spinal ketamine. Eur J Pharmacol, 1991;194: 167-172.

15. Yamakage M, Hirshman CA, Croxton TL - Inhibitory effects of thiopental, ketamine, and propofol on voltage-dependent $\mathrm{Ca}^{++}$ channels in porcine tracheal smooth muscle cells. Anesthesiology, 1996;83:1274-1282.

16. Smith DJ, Bouchal RL, DeSanctis CA et al - Properties of the interaction between ketamine and opiate binding sites in vivo and in vitro. Neuropharmacol, 1987;26:1253-1260.

17. De Kock MD, Eisenach J, Tong $C$ et al - Analgesic doses of intrathecal but not intravenous clonidine increase acetylcholine in cerebrospinal fluid in humans. Anesth Analg, 1997;84: 800-803.

18. Chassard D, Mathon L, Dailler F et al - Extradural clonidine combined with sufentanil and $0.0625 \%$ bupivacaine for analgesia in labor. Br J Anaesth, 1996;77:458-462.

19. Yaksh TL, Pogrel JW, Lee YW et al - Reversal of nerve ligation-induced allodynia by spinal alpha-2 adrenoceptor agonists. J Pharmacol Exp Ther, 1995;272:207-214.

20. Davis KD, Treede RD, Raja SN et al - Topical application of clonidine relieves hyperalgesia in patients with sympathetically maintained pain. Pain, 1991;47:309-317.

21. Rauck RL, Eisenach JC, Jackson K et al - Epidural clonidine treatment for refractory reflex sympathetic dystrophy. Anesthesiology, 1993;79:1163-1169.

22. Khan ZP, Ferguson CN, Jones RM - Alpha-2 and imidazoline receptor agonists. Anesthesia, 1999;54:146-165.

23. Klimscha W, Tong C, Eisenach JC - Intrathecal $\alpha_{2}$-adrenergic agonists stimulate acetylcholine and norepinephrine release from the spinal cord dorsal horn in sheep. Anesthesiology, 1997;87:110-116.

24. Smith MS, Shambra UB, Wilson K et al - Alpha1- adrenergic receptors in human spinal cord: specific localized expression of mRNA encoding alpha 1-adrenergic receptor subtypes at four distinct levels. Mol Brain Res, 1999;63;254-261.

25. Smith MS, Shambra UB, Wilson KH et al - Alpha 2 adrenergic receptors in human spinal cord: specific localized expression of mRNA encoding alpha 2-adrenergic receptor subtypes at four distinct levels. Mol Brain Res, 1995;34:109-117.

26. Ongiocco RR, Richardson CD, Rudner XL et al - Alpha-2 adrenergic receptors in human dorsal root ganglia: predominance of $\alpha_{2 b}$ and $\alpha_{2 c}$ subtype mRNAs. Anesthesiology, 2000;92: 968-976.
27. Nagy I, Woolf CJ - Lignocaine selectivity reduces C fibre-evoked neuronal activity in rat spinal cord in vitro by decreasing $\mathrm{N}$-methyl-D-aspartate and neurokinin receptor-mediated post-synaptic depolarizations; implications for the development of novel centrally acting analgesics. Pain, 1996;64:59-70.

28. Bartolini A, Galli A, Ghelardini C et al - Antinociception induced by systemic administration of local anesthetics depends on a central cholinergic mechanism. Br J Pharmacol, 1987;92: 711-721.

29. Biella G, Sotgiu ML - Central effects of systemic lidocaine mediated by glycine spinal receptors: an iontophoretic study in the rat spinal cord. Brain Res, 1993;603:201-206.

\section{RESUMEN}

Lauretti GR, Rodrigues AM, Gomes JMA, Reis MP - Evaluación Clínica Comparativa entre la Cetamina y la Clonidina por Vía Peridural en el Tratamiento del Dolor Crónico Neuropático

Justificativa y Objetivos - El dolor crónico resulta en respuesta aumentada de los neuronios del cuerno dorsal de la médula espinal, debido a la acción de diferentes mediadores liberados por las terminaciones neuronales, incluyendo agonistas para los receptores N-metil-D-aspartato. Además de las vías ascendentes sensitivas, vías descendentes inhibitorias modulan la sensación de dolor, incluyendo la participación de $\alpha_{2}$-agonistas localizados en los aferentes primarios y en la médula espinal. Este estudio visó evaluar el efecto antinociceptivo de la cetamina (antagonista del receptor $N$-metil-D-aspartato) y de la clonidina ( $\alpha_{2}$-agonista) administrada por vía peridural, en el tratamiento de dolor crónico neuropático.

Método - Veintiséis pacientes adultos con queja de dolor tipo neuropático, no-responsivo a los tratamientos convencionales, fueron de forma prospectiva, aleatoria y duplamente encubierta, divididos en dos grupos. Todos los pacientes hicieron uso de 50-75 mg de amitriptilina por vía oral diariamente. El dolor fue evaluado utilizándose la escala analógica visual (EAV) de $10 \mathrm{~cm}$, en que "cero" correspondió a "ausencia de dolor" y "diez" a "peor dolor imaginable". Un catéter peridural fue introducido en la región lumbar, y las medicaciones por vía peridural fueron administradas en intervalos de 8 horas, durante tres semanas. El Grupo Cetamina recibió $0,1 \mathrm{mg} \cdot \mathrm{kg}^{-1}$ de cetamina sin conservante, seguida de la administración de $30 \mathrm{mg}$ de lidocaína a $1 \%$, en cada aplicación. El Grupo Clonidina recibió $30 \mu \mathrm{g}$ de clonidina sin conservante, seguida de la administración de $30 \mathrm{mg}$ de lidocaína a $1 \%$, en cada aplicación.

Resultados - Veintitrés pacientes hicieron parte de la evaluación final. La administración de cetamina o clonidina por vía peridural, en las dosis propuestas, resultó en analgesia durante todo el período de la manutención del catéter peridural (EAV inicial 8-10 cm versus EAV final 0-3 cm) $(p<0,002)$. Después de la retirada del catéter peridural, los valores numéricos de la EAV se mantuvieron entre $0-3 \mathrm{~cm}$ durante 2 a 5 semanas.

Conclusiones - La administración de cetamina o clonidina por vía peridural resultó en acción antinociceptiva en pacientes con dolor crónico neuropático, no-responsivo a los tratamientos convencionales, representando alternativas eficaces, cuando el tratamiento convencional no obtuvo suceso. 